\title{
HiBi Triangular Optical Nanowire in Suspended-Core Fiber for Sensing Applications
}

\author{
Ricardo M. André ${ }^{\mathrm{a}, \mathrm{b}}$, Martin Becker ${ }^{\mathrm{c}}$, Kay Schuster ${ }^{\mathrm{c}}$, Manfred Rothhardt ${ }^{\mathrm{c}}$, Hartmut Bartelt $\mathrm{t}^{\mathrm{c}}$, Manuel \\ B. Marques ${ }^{\mathrm{a}, \mathrm{b}}$, Orlando Frazão ${ }^{\mathrm{a}}$ \\ ${ }^{a}$ INESC Porto, Rua do Campo Alegre 687, 4169-007 Porto, Portugal; \\ ${ }^{b}$ Departamento de Física da Faculdade de Ciências da Universidade do Porto, Rua do Campo Alegre \\ 687, 4169-007 Porto, Portugal \\ ${ }^{c}$ IPHT, Institute of Photonic Technology, Jena, Albert-Einstein-Str. 9, 07745 Jena, Germany
}

\begin{abstract}
A triangular nanowire is fabricated by tapering a suspended-core fiber and reducing the core size below one micrometer. The triangular nanowire has a high birefringence with an order of magnitude of $10^{-3}$ and when introduced in a fiber loop mirror presents a sinusoidal interference pattern generated by the fast and slow modes of the nanowire. The suspended nanowires were characterized in temperature and strain and enhanced sensitivities were found for both parameters when compared with untapered structures.
\end{abstract}

Keywords: Fiber loop mirror, suspended-core fiber, triangular nanowires, optical fiber sensors, strain and temperature sensing, high birefringence.

\section{INTRODUCTION}

Fiber loop mirror (FLMs) are often used in optical communication and sensing applications [1]. These types of devices are very easy to fabricate and are comprised of a splice between the output ports of an $3 \mathrm{~dB}$ optical fiber coupler. In this embodiment, two waves propagate within the fiber loop in opposite directions, but along the same optical path and constructive interference occurs when the waves recombine in the fiber coupler. Thus all of the light is reflected back to the first input port with reflectivity only limited by the losses in the fiber and of the splices, as well as the coupler. With this property they can be used as high reflectivity mirrors forming a resonant cavity in fiber lasers [2]. The FLM becomes much more interesting when a section of high birefringence fiber is introduced into the fiber loop, becoming a high birefringence fiber loop mirror (HiBi FLM). Independence of input polarization and independence of the spectral response periodicity on the total length of the loop are just the main advantages of these filters [3]. In sensing applications, the HiBi FLMs have been employed for the measurement of strain [4], temperature [5], and the level of liquids [6]. More recently, new FLM-based configurations have been employed for the simultaneous measurement of strain and temperature. Most combine two sensing structures to eliminate temperature cross-sensitivity such as a HiBi fiber with a long period grating [7] or a fiber Bragg grating [8], two different types of HiBi fiber [9] or even two concatenated HiBi FLM [10]. A FLM containing just one sensing structure: section of HiBi PCF has also been demonstrated for temperature independent strain sensing [11].

In this work, a tapered $\mathrm{HiBi}$ suspended-core fiber with a triangular core is characterized in a fiber loop mirror configuration. A two wave interferometer is obtained for the $810 \mathrm{~nm}$ triangular core where only the fast and slow modes persist effectively eliminating the intermodal interference. An increase in temperature and strain sensitivities is achieved due to the properties created by the fabrication of the taper.

*randre@inescporto.pt

Fifth European Workshop on Optical Fibre Sensors, edited by Leszek R. Jaroszewicz, Proc. of SPIE Vol. 8794, $879401 \cdot$ C 2013 SPIE · CCC code: 0277-786X/13/\$18 - doi: 10.1117/12.2025404 


\section{FABRICATION AND CHARACTERIZATION}

Figure 1 illustrates the experimental setup used in this work. It consists of an optical broadband source, a fiber loop mirror (FLM), and an optical spectrum analyzer (OSA) with a maximum resolution of $0.01 \mathrm{~nm}$. The optical source is an erbium-doped broadband source, with a central wavelength of $1550 \mathrm{~nm}$ and a spectral bandwidth of $100 \mathrm{~nm}$. The FLM is formed by a $3 \mathrm{~dB}(2 \times 2)$ optical coupler with low insertion loss, a section of suspended core fiber with approximately 50 $\mathrm{mm}$ in length, and an optical polarization controller. The suspended-core fiber used has an external diameter of $125.6 \mu \mathrm{m}$ and a core diameter of $2.1 \mu \mathrm{m}$. The fiber has three holes that confer the core a triangular shape with high asymmetry and consequently a high birefringence.

On the suspended-core fiber section, tapers with several taper waists were fabricated using a VYTRAN GPX-3000. The VYTRAN (GPX-3000 series) is a glass processing platform that basically heats the fiber to its softening point while applying a tensile force, thus creating a taper.

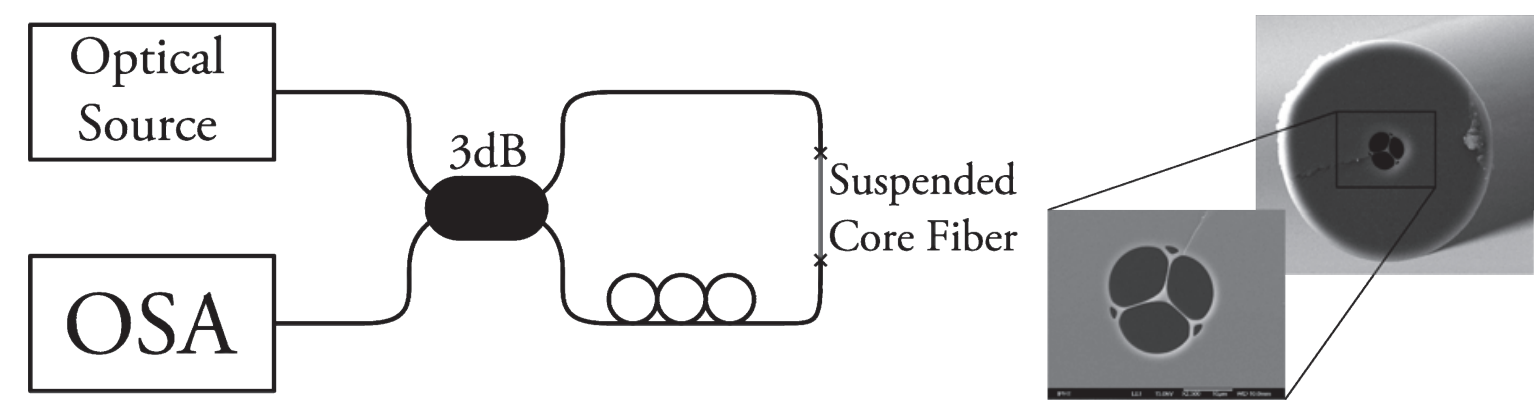

Figure 1 - Fiber loop mirror configuration including a Broadband Optical Source, an Optical Spectrum Analyzer (OSA), a $3 \mathrm{~dB}$ coupler, a polarization controller, and a section of suspended-core fiber (also depicted on the right).

In Figure 2, the spectral responses of the FLM with untapered and tapered SCF are shown. In the untapered case - 2.1 $\mu \mathrm{m}$-wire, (Figure 2a) two interferometers are clearly present: one that results from intermodal interference that presents short-period fringes and one that results from the core's high birefringence that presents long-period fringes. In the tapered case $-810 \mathrm{~nm}$-nanowire, the intermodal interference is significantly reduced and the intermodal fringe visibility is almost completely eliminated. Thus, a spectrum with only the large-period fringes that result from the high birefringence is obtained. At a nanocore of $810 \mathrm{~nm}$, only two modes seem to exist and the sinusoidal response confirms this.
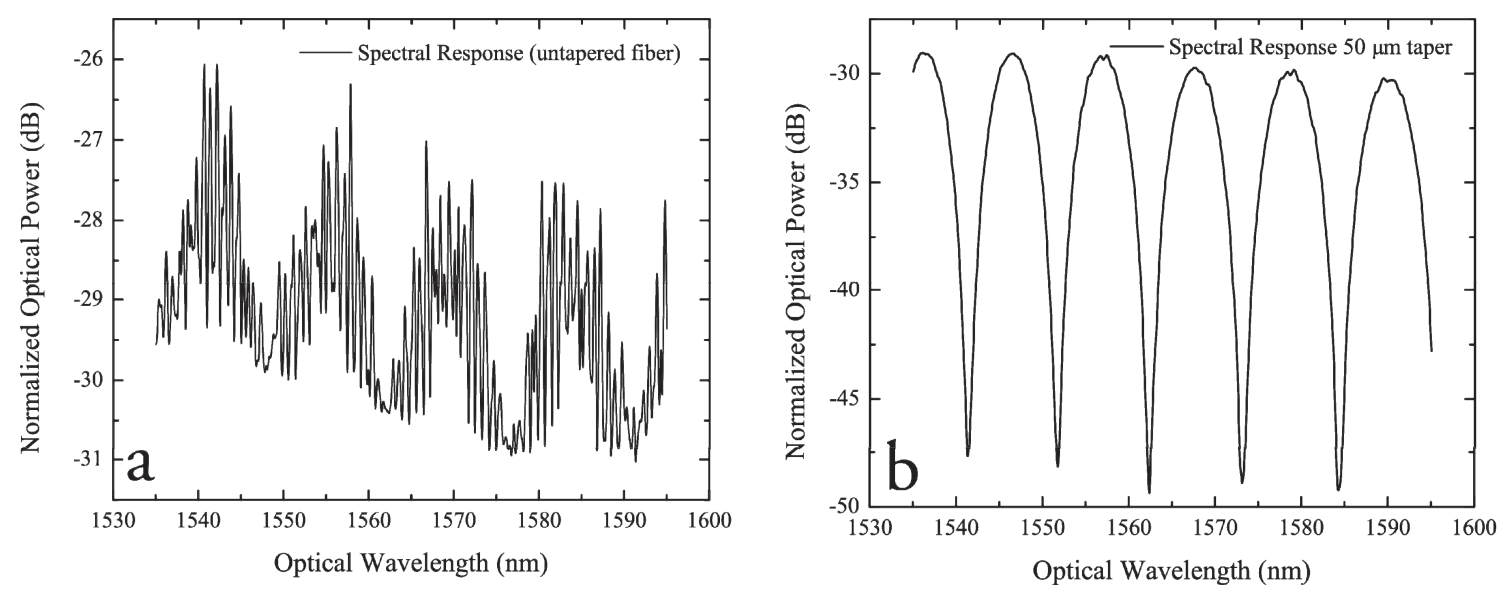

Figure 2 - Spectral responses of the fiber loop mirrors containing: a) a section of untapered SCF; and b) a section of SCF with a $50 \mu \mathrm{m}$-taper. 


\section{RESULTS AND DISCUSSION}

For strain characterization, each sensing head was placed on micrometric translation stages and subjected to controlled stretching. A total length of $0.5 \mathrm{~m}$ was subjected to strain. To determine the wavelength variations, the wavelength change of the main peaks was monitored through an optical spectrum analyzer with a maximum resolution of $0.01 \mathrm{~nm}$ (see Figure 3a). Figure 3b shows an increase in strain sensitivity as the taper waist diameter is decreased. A maximum strain sensitivity of $-2.41 \mathrm{pm} / \mu \varepsilon$ was obtained for the $50 \mu \mathrm{m}$ taper $(810 \mathrm{~nm}$-core). This results in an increase of $236 \%$ relative to the sensitivity of the untapered fiber $(-0.718 \mathrm{pm} / \mu \varepsilon)$.

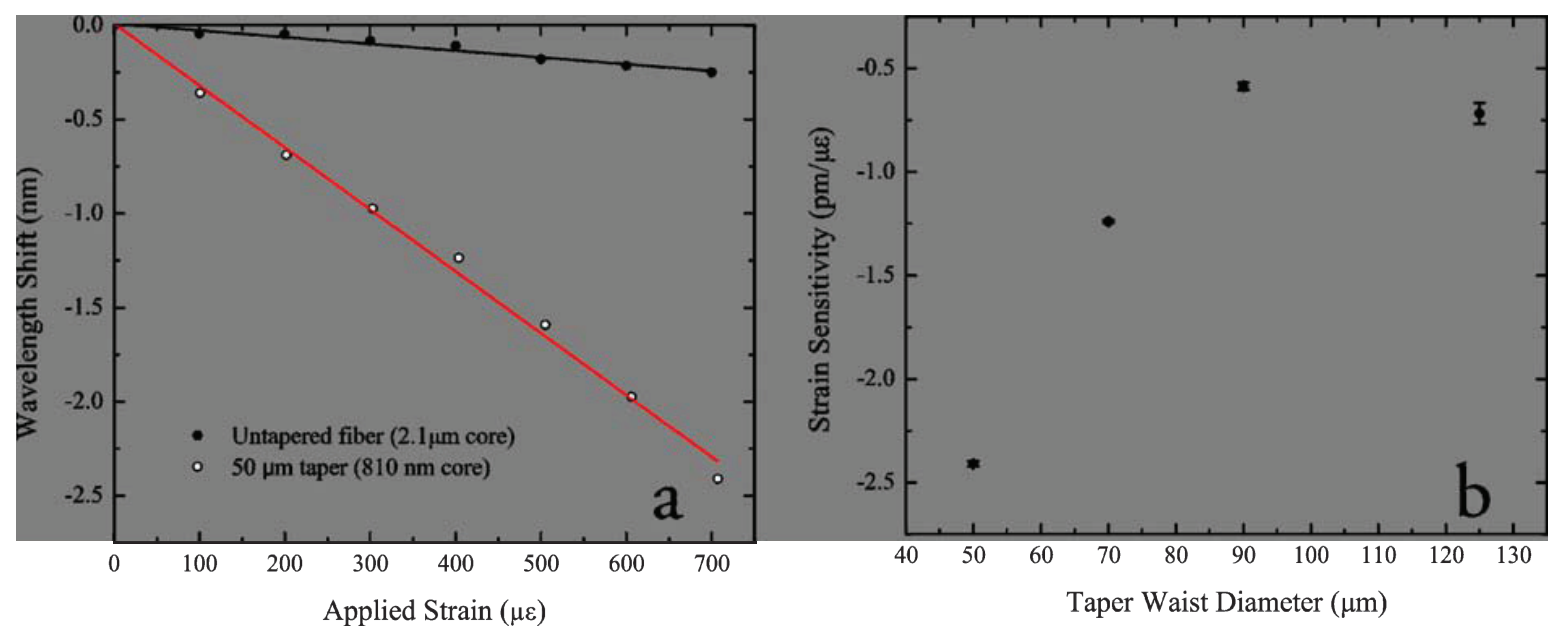

Figure 3 - Strain characterization: a) Wavelength shift with applied strain for untapered and tapered suspended-core fiber $(50 \mu \mathrm{m}$-waist); b) Strain sensitivity as a function of taper waist diameter.

The sensing heads were characterized with respect to temperature variations in the range $35^{\circ} \mathrm{C}$ to $85{ }^{\circ} \mathrm{C}$. In Figure 4 the wavelength shift of the group birefringence fringe pattern is analyzed as a function of the temperature variation for the untapered $2.1 \mu \mathrm{m}$-core $(125 \mu \mathrm{m}$ untapered suspended-core fiber) and the $810 \mathrm{~nm}$-core $(50 \mu \mathrm{m}$ taper $)$. An increase in sensitivity from $-2.1 \mathrm{pm} / \mathrm{K}(2.1 \mu \mathrm{m}$-core $)$ to $-56.2 \mathrm{pm} / \mathrm{K}(810 \mathrm{~nm}$-core $)$ was registered.

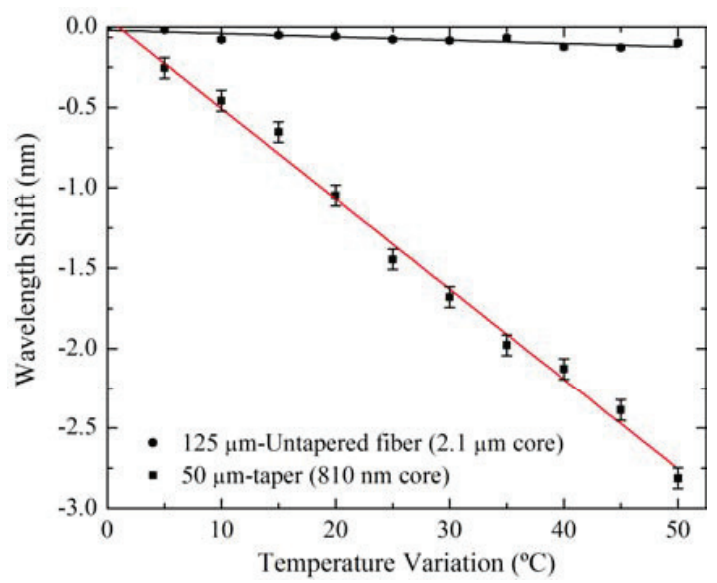

Figure 4 - Temperature characterization: Wavelength shift with temperature variation for untapered and tapered suspendedcore fiber $(50 \mu \mathrm{m}$-waist $)$. 


\section{CONCLUSIONS}

Tapers of several waist diameters were produced on three-hole suspended-core fiber with an original core diameter of 2.1 $\mu \mathrm{m}$. As the core diameter is reduced, the number of guided modes is consequently reduced, and as seen in the spectral responses, the intermodal interference visibility is reduced. At $810 \mathrm{~nm}$, only two modes seem to propagate in the nanowire and the interference pattern is reduced to a two-wave interferometer. Strain sensitivity increases with taper waist reduction. A sensitivity of $-2.41 \mathrm{pm} / \mu \varepsilon$ was obtained for the taper with a core of $810 \mathrm{~nm}, 3.4$ times larger than the untapered fiber's sensitivity. As for temperature, a very high sensitivity of $-56.2 \mathrm{pm} / \mathrm{K}$ for the same taper was obtained, as opposed to the low sensitivity of $-2.1 \mathrm{pm} / \mathrm{K}$ for the untapered case. This is because at lower core diameters temperature increase will induce a larger change in birefringence since the modes are less confined and the contribution of the air's thermo-optic coefficient is higher.

\section{ACKNOWLEDGMENTS}

This work was supported by the ERDF - European Regional Development Fund through the COMPETE Programme (operational programme for competitiveness), by National Funds through the FCT - Fundação para a Ciência e a Tecnologia (Portuguese Foundation for Science and Technology) within project FCOMP-01-0124-FEDER-022701, and by the WOOD project (PTDC/EME-PME/114443/2009). The work of Ricardo André is also supported by FCT through a $\mathrm{PhD}$ Scholarship.

\section{REFERENCES}

[1] D. B. Mortimore, "Fiber loop reflectors,” Journal of Lightwave Technology 6(7), 1217-1224 (1988).

[2] P. Urquhart, "Fiber lasers with loop reflectors," Applied Optics 28(17), 3759-3770 (1989).

[3] Y. Liu, B. Liu, X. Feng, W. Zhang, G. Zhou, S. Yuan, G. Kai, and X. Dong, "High-birefringence fiber loop mirrors and their applications as sensors," Applied Optics 44(12), 2382-2390 (2005).

[4] M. Campbell, G. Zheng, A. S. Holmes-Smith, and P. A. Wallace, "A frequency-modulated continuous wave birefringent fibre-optic strain sensor based on a Sagnac ring configuration," Measurement Science and Technology 10(3), 218-224 (1999).

[5] E. De la Rosa, L. A. Zenteno, A. N. Starodumov, and D. Monzon, "All-fiber absolute temperature sensor using an unbalanced high-birefringence Sagnac loop," Optics Letters 22(7), 481-483 (1997).

[6] D. Bo, Z. Qida, L. Feng, G. Tuan, X. Lifang, L. Shuhong, and G. Hong, "Liquid-level sensor with a highbirefringence-fiber loop mirror," Applied Optics 45(30), 7767-7771 (2006).

[7] O. Frazão, L. M. Marques, S. Santos, J. M. Baptista, and J. L. Santos, "Simultaneous Measurement for Strain and Temperature Based on a Long-Period Grating Combined With a High-Birefringence Fiber Loop Mirror," IEEE Photonics Technology Letters 18(22), 2407-2409 (2006).

[8] D.-P. Zhou, L. Wei, W.-K. Liu, Y. Liu, and J. W. Y. Lit, "Simultaneous measurement for strain and temperature using fiber Bragg gratings and multimode fibers,” Applied Optics 47(10), 1668 (2008).

[9] G. Sun, D. S. Moon, and Y. Chung, "Simultaneous Temperature and Strain Measurement Using Two Types of HighBirefringence Fibers in Sagnac Loop Mirror,” IEEE Photonics Technology Letters 19(24), 2027-2029 (2007).

[10] O. Frazão, J. L. Santos, and J. M. Baptista, "Strain and Temperature Discrimination Using Concatenated HighBirefringence Fiber Loop Mirrors,” IEEE Photonics Technology Letters 19(16), 1260-1262 (2007).

[11] R. M. André, M. B. Marques, P. Roy, and O. Frazão, "Fiber Loop Mirror Using a Small Core Microstructured Fiber for Strain and Temperature Discrimination,” IEEE Photonics Technology Letters 22(15), 1120-1122 (2010). 